\title{
Neglected Bilateral Rupture of the Achilles Tendon: A Case Report and Review of the Literature
}

\author{
J alal $Y^{1 *}$, Zaddoug $\mathbf{O}^{1}$, Soukaina $\mathrm{Z}^{2}$, Bah $\mathrm{A}^{1}$, Zine $\mathrm{A}^{1}$ \\ and J aafar $\mathbf{A}^{\mathbf{1}}$ \\ ${ }^{1}$ Department of Orthopedic Surgery and Traumatology, \\ Military Hospital Mohammed V, Rabat, Morocco \\ ${ }^{2}$ Department of Radiology, Military Hospital Mohamed V, \\ Faculty of Medicine and Pharmacy of Rabat, Morocco \\ *Correspondling author: Youssef J alal, Department of \\ Orthopedic Surgery and Traumatology, Military Hospital \\ Mohamed V, Faculty of Medicine and Pharmacy, Rabat, \\ Morocco
}

Received: October 06, 2017; Accepted: November 07, 2017; Published: November 14, 2017

\begin{abstract}
Simultaneous bilateral rupture of the Achilles tendon is uncommon, often associated with systemic diseases and a long history of corticosteroid consumption. The author's report a rare case of a 65-year old man with known Chronic Obstructive Airway Disease (COAD) for which he takes regularly beclomethasone, salmeterol and salbutamol inhalers, for last ten years, admitted for neglected and spontaneous bilateral rupture of the Achilles tendon. The management of a condition is still controversial. Our preference was for open surgical treatment according to Bosworth technique with augmentation by plantaris tendon. Six months after surgery, the patient was able to perform bilateral heel rise without difficulty.
\end{abstract}

Keywords: Achille tendon; Chronic rupture; Corticosteroid consumption

\section{Introduction}

Ruptures of Achilles tendon are common and serious injuries, however bilateral forms are relatively rare with an incidence of about $1 \%$ only [1]. This condition is usually present in patients at the third to fourth decades of life with a marked Male dominance. Usually occurred Spontaneously, bilateral rupture of Achilles tendon are frequently described in subjects with systemic diseases, who are receiving long-term corticoid treatment [2], and recently in patients under fluoroquinolone antibiotic treatment [3]. Neglected rupture of the Achilles tendon is when the patients are untreated for 4 weeks after the injury. Which is a challenge in management? We report a case of a neglected bilateral rupture of the Achilles tendon in a Chronic Obstructive Airway Disease (COAD) patient.

\section{Observation}

A 65-year old man with known diabetes treated by metformin (2000 mg daily) and Chronic Obstructive Airway Disease (COAD) for last ten years for which he takes regularly beclomethasone, salmeterol and salbutamol inhalers. The Patient was referred to our department for inability to walk unaided since 3 months. In his history, the patient complained that while he tried to push a heavy object, he felt a sharp pain in the left heel simultaneously later he had similar pain in the other heel, and he was unable to ambulate. $\mathrm{He}$ was presented to the emergency department for bilateral pain ankle and bruising. A diagnosis of bilateral ankle sprain was retained. Not improved, the patient consulted to our department 3 months later. On physical examination the patient presented with partial limitation of plantar flexion of the foot, and a positive Thompson test findings bilaterally. On the right side, a gap was palpable in the Achille tendon approximately $6 \mathrm{~cm}$ proximal to the insertion. On the left side, there was obvious palpable defect $3 \mathrm{~cm}$ to the distal insertion (Figure 1). Radiographs did not show any bone's fracture or avulsion (Figure 2). A final diagnosis of neglected bilateral rupture of the Achille tendon was made by ultrasound.

After adequate information on the surgical options, the patient was admitted in operating room. He was positioned prone on the table and an air tourniquet was applied on the proximal right thigh, then on the left one. Through a $12 \mathrm{~cm}$ longitudinal incision arising the medial border of the Achilles tendon, a careful dissection of the subcutaneous tissue, and release of the peritendinous adhesions were made.Thus, the tendon stumps are identified between which an extensive fibrose tissue was interposed (Figure $3 \mathrm{~A}, 3 \mathrm{~B}$ ). This tissue was debrided on both ends of the rupture site. After measuring the gap, a $2 \mathrm{~cm}$ wide strip was cut from the gastrocnemius muscle and sutured to the distal stump of the Achilles (Figure 4A,4B). Then the plantaris tendon was harvested, rolled up and placed around repair site for augmentation as was described by Chigot. The wound was then irrigated and closed (Figure 7). A similar surgical procedure was made for the left side. The patient was immobilized in both posterior splint with minimal plantar flexion for 3 weeks (Figure 8), followed by the neutral position for another 3 weeks and early active mobilization exercises was performed.

The patient was seen in follow-up 2 weeks later for routine evaluation, and the exam revealed a minimal wound breakdown succefully managed by a controlled wound healing with antibiotic coverage (Figure 5).

At his 6 months postoperative, the patient could perform bilateral

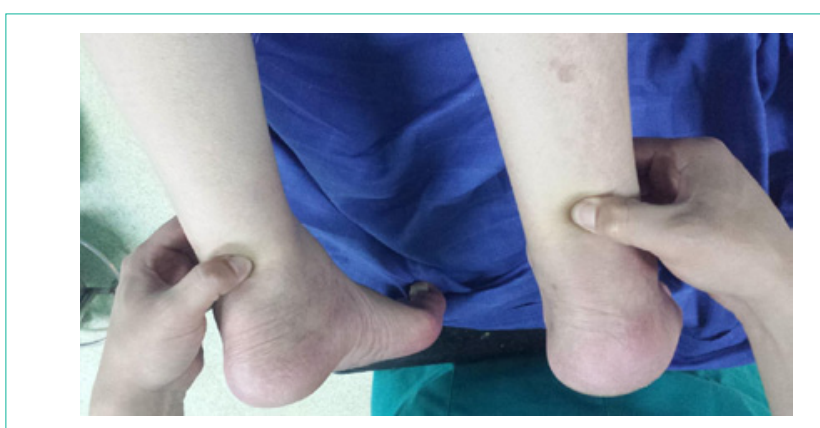

Figure 1: Image showing in the right ankle a gap in the Achille tendon approximately $6 \mathrm{~cm}$ proximal to the insertion, and in the left side a gap $3 \mathrm{~cm}$ to the distal insertion.
Austin J Orthopade \& Rheumatol - Volume 4 Issue 3 - 2017 ISSN: 2472-369X | www.austinpublishinggroup.com Jalal et al. (C) All rights are reserved
Citation: Jalal Y, Zaddoug O, Soukaina Z, Bah A, Zine A and Jaafar A. Neglected Bilateral Rupture of the Achilles Tendon: A Case Report and Review of the Literature. Austin J Orthopade \& Rheumatol. 2017; 4(3): 1058. 


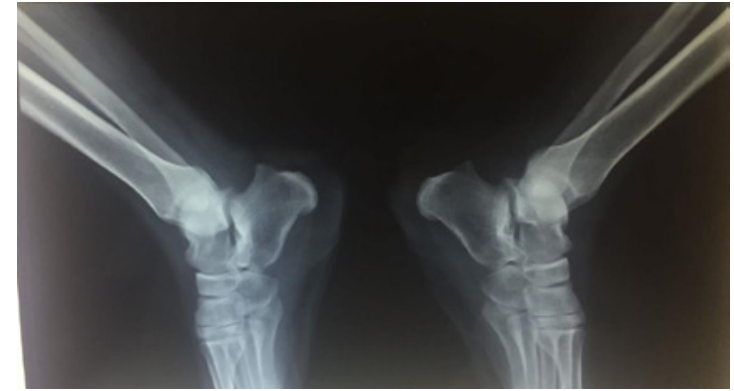

Figure 2: X-rays showing no bone alterations.

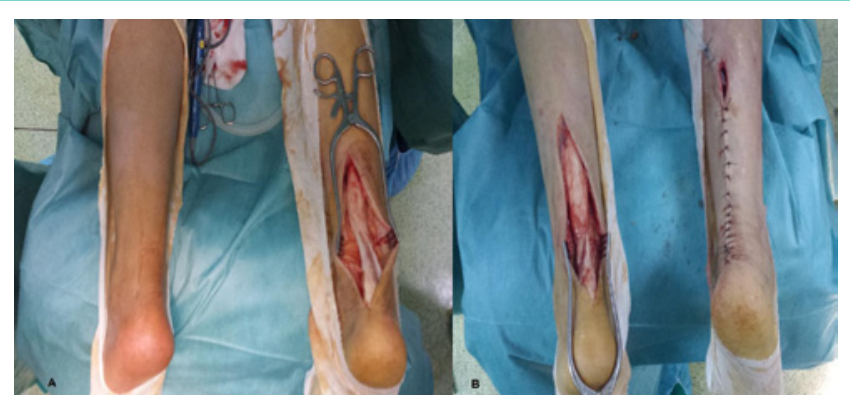

Figure 3: Intraoperative photo showing a gap with interposed scar tissue. A $=$ right ankle, $\mathrm{B}=$ left ankle.
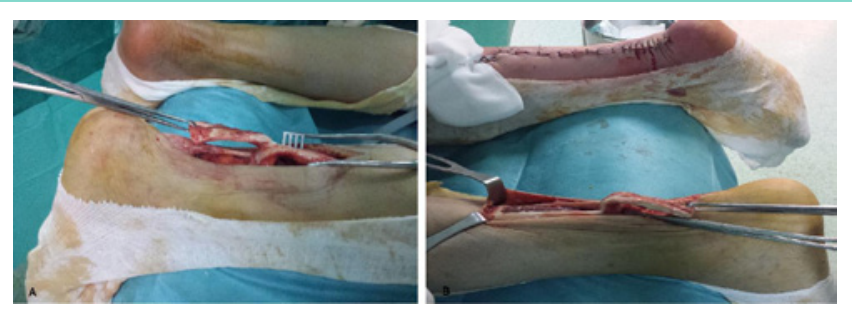

Figure 4: Intra operatively, a proximal portion of the gastrosoleal complex has been mobilized and sutured to the distal stump of the Achilles. . $A=$ right ankle, $\mathrm{B}=$ left ankle.

heel rise without difficulty. For last 3 years, he remains free of any tendon disorder.

\section{Discussion}

Despite it is the largest and strongest tendon in the human body, Achilles tendon ruptures are the most common tendon rupture of the lower extremity and may account for up to $40 \%$ of all tendon injuries $[4,5]$. However, bilateral rupture is uncommon and usually occurs in patients receiving long-term corticoid treatment. The effects of glucocorticoids on cell, and tissue are highly specific. Wong et al. postulated that the suppressed cellular activity and collagen synthesis in human tenocytesmay lead to disturbed tendon structure, thus predisposing the tendon to subsequent spontaneous rupture [6].

Clinically, patient with bilateral rupture of Achilles tendon is usually unable to stand on the toes and his plantar flexion is limited with a palpable gape on physical examination. However, 20-25\% cases are misleading due to scare tissue [7]. In this condition, careful digital palpation completed by Ultrasound as well as MRI is useful tools in confirming the clinical diagnosis. Undiagnosed during 4 weeks, rupture is classified as neglected.

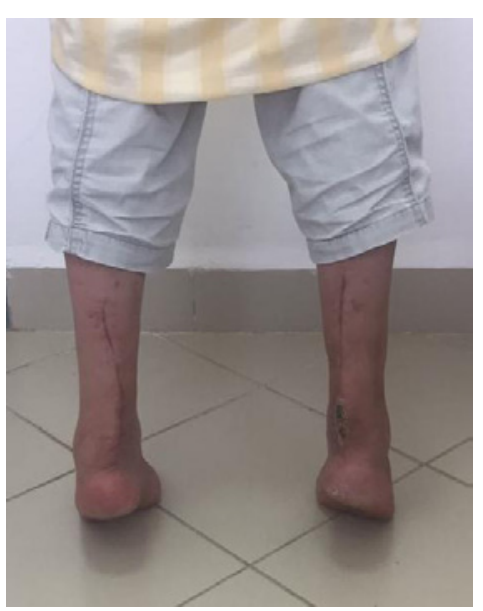

Figure 5: Two months post-operative photo shows superficial wound breakdown on the right ankle during the healing phase. To note that at his second month post-operative, the patient begins to perform a bilateral single heel rise.

Management of neglected bilateral rupture of the Achilles tendon represents a special challenge. Various methods have been described to treat those injuries, but there is no clearcut superiority between them.

Conservative treatment is an option for Achilles tendon tear. It is aim to restore and maintain alignment and contact between the two ends of the ruptured Achilles tendon to facilitate healing by braces, splints or Orthotics which provided good outcome and the risk of wound breakdown is eliminated. Weber et al. postulated that conservative management with an equinus ankle cast and boot for 12 weeks was as effective as surgical treatment in return to sports and ultimate strength as operative treatment [8]. However, this attitude may lead to stiffness and weakness due to muscle atrophy, with a rates of re-rupture of $9,6 \%$.

Regarding the surgical treatment, various surgical techniques among them local tendon transfer, free tissue transfer, and synthetic augmentation are more popular. However, there is no currently consensus on which technique may lead to better outcomes. Several authors suggest that surgical option is the treatment of choice in neglected bilateral rupture of the Achilles tendon. Khan et al. [9] performed retrospective met analysis of twelve randomized controlled and concluded that open operative treatment was associated with a lower risk of re-rupture compared with conservative management (relative risk, 0.27), and plantar flexion strength was higher in the operative group (75\% of uninjured side) than the conservative group (65\% of uninjured). Although it provides increased strength of tendon and allows earlier motion, surgical treatment is associated with a large number of complications, such as wound breakdown and infection rates.

In this article, the patient had a history of steroid consumption, and he presented with heel pain and loss of plantar flexion since 3 months. Intraoperative, the quality of tendon stump is insufficient, and reconstruction was required. We used a Bosworth technique with augmentation by plantaris tendon. During the course of therapy and recovery, we observed superficial wound breakdown successfully managed by a controlled wound healing with good antibiotic 
coverage.

Early rehabilitation is advocated by most of the authors [10]. Whether the treatment is surgical or conservative, the rehabilitation progresses slowly into strengthening, gait and balancing activities and individual patients will progress at different rates depending on preinjury function, the extent of the injury and the commitment to the rehabilitation program.

\section{Conclusion}

Achilles tendon rupture is a serious complication of corticosteroid therapy. It frequently occurred in elderly patients. Currently, there is quite controversy about suitable option to manage those injuries. However, surgical repair is considered the treatment of choice. Several surgical techniques have been described to repair neglected Achilles tendon ruptures and they varied between classic options including augmentation, local tendon transfer, and the new ones with synthetic augmentation, and free tissue transfer .recently, biologic adjuncts, such as Platelet-Rich Plasma (PRP) and bone marrow-derived stem cells, have been used in efforts to optimize postoperative tendon healing, they have yet to show substantial differences in outcome.

\section{References}

1. Rao SK, Navadgi BC, Vasdev A. Bilateral spontaneous rupture of Achilles tendons: A Case Report. J Orthop Surg (Hong Kong). 2005, 13: 178-180.
2. Wanivenhaus A. Tendon ruptures in rheumatic patients. Z Rheumatol. 2007; 66: 36-40.

3. Sode J, Obel N, Hallas J, Lassen A. Use of fluorquinolone and risk of Achilles' tendon rupture: a population-based cohort study. Eur J ClinPharmacol. 2007; 63: 499-503.

4. Leppilahti J, Orava S. Total Achilles tendon rupture: A Review. Sports Med. 1998; 25: 79-100.

5. Habusta SF. Bilateral simultaneous rupture of the Achilles tendon. A rare traumatic injury. Clin Orthop 1995; 320: 231-234.

6. Wong MW, Tang YN, Lee KM, Fu SC, Chan BP, Chan KM. Effect of Dexamethasone on Human Tenocytes and its Reversibility by PDGFBB. J Bone Joint Surg. 2003; 85: 1914-1920.

7. Maffulli N, Dymond NP, Capasso G. Ultrasonographic findings in subcutaneous rupture of Achilles tendon. Journal of Sports Medicine and Physical Fitness. 1989; 29: 365-368.

8. Weber M, Niemann M, Lanz R, Muller T. Nonoperative treatment of acute rupture of the achilles tendon: results of a new protocol and comparison with operative treatment. Am J Sports Med. 2003; 31: 685-691.

9. Khan RJ, Fick D, Keogh A, Crawford J, Brammar T, Parker M. Treatment of acute achilles tendon ruptures. A meta-analysis of randomized, controlled trials. J Bone Joint Surg Am. 2005; 87: 2202-2210.

10. Talbot JC, Williams GT, Bismil Q, Shaw DL, Schilders E. Results of accelerated postoperative rehabilitation using novel "suture frame" repair of Achilles tendon rupture. J Foot Ankle Surg. 2012; 51:147-151.
Austin J Orthopade \& Rheumatol - Volume 4 Issue 3 - 2017 ISSN: 2472-369X | www.austinpublishing group.com Jalal et al. (C) All rights are reserved
Citation: Jalal Y, Zaddoug O, Soukaina Z, Bah A, Zine A and Jaafar A. Neglected Bilateral Rupture of the Achilles Tendon: A Case Report and Review of the Literature. Austin J Orthopade \& Rheumatol. 2017; 4(3): 1058 